\title{
Valoración sociojurídica del cuidador de la persona mayor
}

\author{
Partner-Artificial Valuation to the Grown-Up's Caretaker \\ Avaliação sócio-jurídica do cuidador de idosos
}

\author{
Celín Pérez NÁJERA* \\ LISSET DÍAZ ECHEMENDÍA ${ }^{* *}$ \\ Bertha Rosana Llontop CASTAÑEDA ${ }^{* *}$
}

FECHA DE RECEPCIÓN: 8 DE MAYO DE 2020. FeCHA DE APROBACIÓN: 13 DE NOVIEMBRe DE 2020

Doi: https://doi.org/10.12804/revistas.urosario.edu.co/sociojuridicos/a.9082

Para citar este artículo: Pérez Nájera, C., Díaz Echemendía, L., \& Llontop Castañeda, B. R. (2021). Valoración sociojurídica del cuidador de la persona mayor. Revista Estudios Socio-Jurídicos, 23(2), 131-158. https://doi.org/10.12804/ revistas.urosario.edu.co/sociojuridicos/a.9082

\section{RESUMEN}

El objetivo principal de este artículo es proporcionar un material bibliográfico actualizado sobre el tema del cuidador de la persona mayor desde las ópticas criminológica y victimo-criminológica, con énfasis en la atención de este grupo etáreo en Cuba y su proyección para enfrentar la pandemia del coronavirus. Utilizamos como método de investigación un enfoque dialéctico-materialista de la realidad, que posibilitó el engranaje de toda la investigación, teniendo en cuenta el carácter teórico-doctrinal del texto, lo que determinó que esta se enmarcara en torno a los métodos: histórico-lógico, analítico-sintético, inductivo-deductivo, teórico-jurídico y sociológico. Al concebir los resultados como esenciales para una fundamentación teórica desde la criminología sobre la conceptualización de esta figura, la determinación de los elementos configurativos del cuidador de la persona mayor, valoramos también la atención del Estado a este grupo etáreo ante la introducción y propagación de la COVID-19. Dentro de ello

* Doctora en Ciencias Jurídicas y Especialista en Derecho Penal. Profesora Titular de la Facultad de Ciencias Sociales y Humanísticas. Rectora de la Universidad de Ciego de Ávila Máximo Gómez Báez. Cuba. Correo electrónico: celinpn1973@gmail.com ORCID: https:// orcid.org/0000-0002-8964-6427

** Licenciada en Derecho. Abogada de la Organización de Bufetes Colectivos del municipio de Ciego de Ávila. Provincia de Ciego de Ávila. Cuba. ORCID: https://orcid.org/00000002-6058-762X

*** Licenciada en Marketing y Dirección de Empresas. Maestría en Administración de Negocios. Jefa de Relaciones Internacionales de la Universidad Cesar Vallejo en Chiclayo. Correo electrónico: rosanillontop@gmail.com ORCID: https://orcid.org/0000-0002-1209-0002 
proponemos juicios organizativos de las variantes en que puede presentarse, así como el análisis de su regulación jurídica en las legislaciones nacionales.

Palabras claves: persona mayor; cuidador; envejecimiento; pandemia; vulnerable.

\begin{abstract}
The essential objective of this article is to provide an up-to-date bibliographical material on elders' caretakers from the optic of criminology and crime victim with emphasis on the attention of this group in Cuba and its projection to face the coronavirus pandemic. We use as investigation model a dialectical-materialistic approach of reality that facilitated the engagement of the whole investigation, keeping in mind the theoretical-doctrinal character of the text, which determined that the method was framed around the methods: historical-logical, analytic-synthetic, inductive-deductive, theoretical-juridical and sociological. Conceiving as essential results a theoretical foundation from the criminology on the conceptualization of this figure, the determination of the elements configurative of the elderly' caretakers, we also value the attention from the State to this group ethereal before the introduction and propagation of the COVID-19 with a look from Cuba and inside we propose it organizational trials of the variants in that it can be presented, as well as the analysis of their artificial regulation in the national legislations.
\end{abstract}

Keywords: grown-up; caretaker; aging; pandemic; vulnerable.

\title{
RESUMO
}

Disponibilizar um material bibliográfico atualizado sobre a temática do cuidador de idosos na perspectiva criminológica e vítima-criminológica é o objetivo central deste artigo. Com ênfase no atendimento dessa faixa etária em Cuba e sua projeção para o enfrentamento da pandemia do Coronavírus. Utilizamos como Metodologia Investigativa uma abordagem dialético-materialista da realidade, que possibilitou o engrenamento de toda a pesquisa, levando em conta a natureza teórico-doutrinária do texto, o que determinou que o método se enquadrasse nos métodos: histórico-lógico, analítico-sintético, indutivo-dedutivo, teórico-jurídico e sociológico. Concebendo como resultados essenciais uma fundamentação teórica da Criminologia sobre a conceituação desta figura, a determinação dos elementos configurativos do cuidador do idoso, avaliamos também a atenção do Estado para com esta faixa etária perante a introdução e propagação do COVID-19 sob uma visão cubana, e dentro dela propomos julgamentos organizacionais das variações em que pode ser apresentada, bem como a análise de sua regulamentação legal na legislação nacional.

Palavras-chave: idoso; cuidador; envelhecimento; pandemia; vulnerável. 


\section{Introducción}

El envejecimiento afecta a la población a escala mundial, todos los países marchan hacia sociedades más envejecidas, aunque a diferentes ritmos; o sea, unos más acelerados que otros, pero de un modo inexorable se producirá la transición demográfica, lo que obliga a la familia, al Estado y a la estructura política y económica a abordar la figura de la vejez como un fenómeno sin precedente que requiere un enfrentamiento multisectorial, con una visión intradisciplinar, diversificada, especializada e integral.

La población mundial continúa creciendo de una manera alarmante. En la actualidad somos 7000 millones de personas y para el 2050 se pronostica la cifra de 9500 millones de habitantes. Preocupa entonces el análisis de las estadísticas en las que se predice que el número de personas mayores de 60 años en el planeta se triplique, de 605 millones a casi 2000 millones en el 2050. Cuba, con un 20,8\% de su población mayor de 60 años, es uno de los países más envejecidos de América Latina y está previsto que sea en el 2030 uno de los más envejecidos del mundo con el 30,1 \% de esta población (Organización Mundial de la Salud [OMs], 2018, p. 2; Oficina Nacional de Estadísticas e Información, 2018, p. 12).

En este caso, el envejecimiento poblacional se produce por la acción combinada de dos connotadas transformaciones sociales que poseen una fuerte relación entre sí: la demográfica y la epidemiológica o de salud. Definidas brevemente, la primera se refiere a los cambios en las estructuras de la población, debido esencialmente a las bajas tasas de fertilidad y de mortalidad. La segunda responde a los enormes avances en materia de salud humana, progresos caracterizados por la existencia de menor incidencia, prevalencia y letalidad de las enfermedades crónicas degenerativas e incapacitantes.

Para la investigación se utilizaron los métodos histórico-lógico, analítico-sintético, inductivo-deductivo, teórico-jurídico y sociológico. Estas valoraciones generalizadoras y sistematizadoras tienen un enfoque modelativo y sistémico-estructural.

Cuando analizamos los fenómenos y procesos que se producen en la sociedad, el más complejo de todos los sistemas existentes, es necesario 
considerar los rasgos generales de la causalidad y lo específico de la vida social. Lo específico indica que todas las regularidades sociales se realizan a través de la actividad de las personas. Se puede por tanto relacionar una serie de particularidades de los vínculos causales en el medio social (Navarrete, 2004, p. 21), desde esa óptica, la criminología reviste una especial importancia en el estudio del contexto social y del grupo de riesgo, debido a sus características de sensibilidad y fragilidad por la edad. Su estudio y atención se potencian desde una visión victimocriminológica por ser considerado un grupo victimal de la realidad social.

Es durante esta etapa de la vida donde el ser humano se torna más vulnerable y necesitado de cuidados, hecho que nos obliga a enfrentar con más premura la situación actual de los cuidadores en el tratamiento de la persona mayor (Organización de Estados Americanos, 2015, p. 4). Nos encontramos viviendo una de las realidades más difíciles y con alto costo humano que ha tenido la historia: la pandemia de enfermedad por coronavirus que se identificó por primera vez en diciembre de 2019 en Wuhan, República Popular China, al presentarse un grupo de personas con neumonía de etiología desconocida. Su nivel de expansión y afectación en el mundo ha sido inimaginable, se han informado 3575412 millones de contagios en más de 184 países, con más de 253717 muertes y 1181403 personas recuperadas. Cuba no ha quedado exenta, se muestra una cifra de 1685 pacientes confirmados con 69 fallecimientos y 954 recuperados (OMS, 2018, p. 3; Ministerio de Salud Pública de la República de Cuba, 2020b, párr. 6).

A estas alturas, ya nadie ignora que la pandemia no es solo una crisis sanitaria. Es lo que las ciencias sociales califican de "hecho social total", en el sentido de que convulsa el conjunto de las relaciones sociales, y conmociona a la totalidad de los actores, instituciones y valores. La humanidad está viviendo con miedo, sufrimiento y perplejidad una experiencia inaugural. (...) Nadie sabe interpretar y clarificar este extraño momento de tanta opacidad. (...) Angustiados, los ciudadanos vuelven sus ojos hacia la ciencia y los científicos, como antaño hacía la religión, implorando el descubrimiento de una vacuna salvadora, (...) se busca también refugio y protección en el Estado (Ramonet, 2020, p. 12). 
Las personas mayores plantean desafíos específicos al constituirse como una población heterogénea en términos de salud, discapacidad y demanda de servicios, lo cual insta y reclama atención priorizada y especializada. Debemos tener en cuenta que, junto al progresivo proceso de envejecimiento, aumentan los índices de dependencia de estas personas, por ello, las salidas a este problema demográfico tan importante transitan por el desarrollo atemperado y evolutivo de las estructuras sociales, económicas y culturales.

\section{Concepciones doctrinales del cuidador}

Para abordar la figura del cuidador analizamos su carácter multidimensional, el cual comprende un conjunto de elementos integradores de diferentes dimensiones centradas en la atención a la persona, relacionadas con las características sociales, familiares y culturales relativas al bienestar de los individuos vulnerables.

Debemos partir de evaluar la distinción entre los términos cuidar y asistir, con el objetivo de comprender más fácilmente los conceptos que se expresan. Asistir y cuidar pueden ser sinónimos de acuerdo con el diccionario; sin embargo, el primero significa acompañar, servir y socorrer, mientras el segundo se refiere a las concepciones de atender, esmerarse y mimar (Alvero, 1978, pp. 97 y 215).

En la práctica, la diferencia pasa por la palabra amor, el que asiste sirve, el que cuida sirve con amor, ternura dedicación y pasión; una madre no asiste a su hijo, una madre cuida a su hijo (Pérez, 2016, p. 2). El cuidar implica atención personal, vigilancia y acompañamiento, brindar cariño, entrega, comprensión, apoyo y ayuda en las labores que resultan ser un poco complejas para la persona necesitada, es hacerse cargo de una persona y velar por su bienestar.

El término se define también como una relación y un proceso:

no es la mera ejecución de tareas o la realización de procedimientos prescritos por un médico, el objetivo de los cuidados va más allá de la enfermedad, es una clase de relación constituida por una disposición genuina para con el otro, reciprocidad y el compromiso de promover 
el bienestar del otro. El cuidado es un trabajo de amor y con ello se manifiesta su dualidad: el amor o interés emocional por la persona que recibe los cuidados y el aspecto práctico de cuidar a otro (de la Cuesta, 2013, p. 34).

Por su parte se destaca que el cuidar surge con la propia aparición del ser humano y presenta dos formas de significado. Por una parte, el cuidado del ser humano a sí mismo y, por otra, el cuidado del otro. La segunda se define como un tipo de relación social específica, basada en afecto, servicio, cariño y trabajo, que se realiza a través de la capacidad de cuidado informal de mujeres, madres, hijas, vecindad o amistad, o de cuidado formal provista por enfermeras, maestras y trabajadoras sociales, entre otras (Guimaráes, 2015, p. 86). Esta última conceptualización es la que acogemos en nuestra investigación para abordar a la persona destinada al cuidado de los gerontes.

El cuidador es en no pocas ocasiones el recurso, instrumento y medio por el cual se proveen cuidados específicos y muchas veces especializados a los enfermos crónicos; es decir, en ellos se deposita o descansa el compromiso de preservar la vida de otro. Se definen también como "aquella persona que asiste o cuida a otra afectada de cualquier tipo de discapacidad, minusvalía o incapacidad, que le dificulta o impide el desarrollo normal de sus actividades vitales o de sus relaciones sociales" (Cruz \& Ostiguin, 2016, p. 3; de los Reyes, 2008, p. 12).

Proporcionar ayuda es una buena forma en que las personas necesitadas sientan que sus carencias físicas, sociales y afectivas están resueltas; para los cuidadores implica una dedicación importante de tiempo y energía, que implica tareas que pueden no ser cómodas y agradables, cuando se cuida suele darse más de lo que se recibe.

\section{Clasificaciones del cuidador de la persona mayor}

La figura del cuidador de la persona mayor ha sido abordada desde diferentes perfiles investigativos, ya sea desde lo clínico, lo sociológico o lo jurídico. En cada uno de ellos encontramos una amplia gama de clasificaciones: el cuidador formal, el informal, los cuidadores principales 
o primarios, los secundarios, los cuidadores empíricos, los profesionales, los domiciliarios, así como los cuidadores por beneficios. Al analizar la variedad de prototipos de estos cuidadores y en aras de conformar una agrupación clasificatoria concebimos dos grandes grupos de cuidadores: cuidadores formales y cuidadores informales.

Los cuidadores formales están provistos por grupos u organizaciones que no están unidos por lazos de parentesco o amistad con la persona que recibe los cuidados, estos cuidadores reciben un beneficio patrimonial por la acción de cuidar y son ofrecidos en instituciones. Dentro de ellos contamos con los cuidadores empíricos y profesionales. Por su parte, los cuidadores informales se presentan con una amplia clasificación, este tipo de cuidadores se concibe como no institucional, pueden recibir o no remuneración, generalmente hay una relación de parentesco con el paciente. Está constituido por cuidadores independientes, miembros de la familia que trabajan por su cuenta para cuidar o personas que buscan en esa vía un empleo, este se considera constituido por las actividades de provisión de servicios personales en el ámbito doméstico a sujetos con necesidades especiales (Gallardo \& Rojas, 2016, p. 17). Dentro de los cuidadores informales encontramos los cuidadores domiciliarios, los primarios, secundarios, empíricos y por beneficio. Según estas clasificaciones la mayor parte de los cuidadores de personas que prestan este tipo de ayuda informal son los hijos, cónyuges y nueras, tal y como analizaremos más adelante.

Resumiendo lo anterior, clasificamos la figura del cuidador desde dos ópticas diferentes, el cuidado informal fundado en una relación social basada en afecto, cariño y servicio que se realiza a través de una relación de parentesco, vecindad o amistad, y el cuidado formal provisto por asistentes o enfermeras, entre otras.

\section{La atención del Estado a las personas mayores ante la introducción y propagación de la COVID-19: una mirada desde Cuba}

La impaciencia que se vive en el mundo nos obliga a una mirada diferente de las sociedades desde la política, la economía, los sistemas 
de salud, las estructuras sociales, el uso de las tecnologías y la desprotección de los grupos más vulnerables, entre otros. Esta situación debe servir de experiencia para reformular el contrato social y avanzar hacia más altos niveles de solidaridad comunitaria y mayor integración social. Cualquier respuesta postpandemia debería apoyarse en los principios de una economía verdaderamente regenerativa, basada en el cuidado y la reparación (Ramonet, 2020, p. 12). Indudablemente, defendemos un modelo global más humano y solidario.

Se ha demostrado que las personas mayores tienen un mayor riesgo de agravarse al contraer COVID-19, esencialmente, porque a medida que las personas envejecen su sistema inmunológico cambia, lo que dificulta que su cuerpo combata enfermedades e infecciones, por lo que tienen más probabilidades de presentar problemas de salud subyacentes, lo cual dificulta su recuperación. La edad aumenta el riesgo de que el sistema respiratorio o los pulmones se cierren cuando una persona mayor se enferma.

En la cotidianidad, la desconfianza e incertidumbre han crecido. Algunos fanáticos ultraliberales no han tardado en reclamar sin tapujos la eliminación maltusiana de los más débiles. El vicegobernador de Estados Unidos, Dan Patrick, declaró que los abuelos deberían sacrificarse y dejarse morir para salvar la economía. En Holanda, el primer ministro ultraliberal, Mark Rutte, apuesta por la inmunidad de rebaño, el jefe de epidemiología del Centro Médico de la Universidad de Leiden, Frits Rosendaal, declaró que no se deben admitir en las unidades de cuidados intensivos a personas demasiado viejas. La COVID-19 no distingue, pero las sociedades desigualitarias sí (Ramonet, 2020, p. 12).

Al escuchar estos criterios, se pueden evaluar cuáles son las medidas de atención a este grupo vulnerable. Para ello debemos contar con un sistema de prevención legitimado por la voluntad política y la activa participación de los ciudadanos donde

los Estados que no han desarrollado políticas adecuadas para apoyar a la persona y al cuidador, (...) tengan la obligación de garantizarle todos sus derechos y eso incluye darles apoyo para que tengan 
actividades durante el día, para que puedan tomar sus decisiones y para que puedan seguir participando (Devandas, 2019, párr. 4).

Independientemente se "están emprendiendo medidas sanitarias para reducir los contagios y de contención social en favor de los grupos más vulnerables para tratar de contrarrestar los efectos económicos negativos de la pandemia. También [se] están tomando medidas económicas, fiscales y monetarias" (Hadar, 2020, párr. 7). No debemos perder de vista que el efecto positivo que debe existir en el grupo etáreo no se percibe, falta la prevención intencionada estructural, que incluye un grupo de acciones para su integración social.

La sociedad debe ser solidaria y proteger mejor a las personas mayores, uno de los grupos más afectados por la pandemia; las medidas de distanciamiento social no deben convertirse en exclusión. Los protocolos de prueba deben basarse en las necesidades de salud y deben descalificar los criterios que no sean médicos, como la edad (Kornfeld-Matte, 2020, párr. 12; Adhanom, 2020, parr. 7).

La mayoría de los Estados no se prepararon desde sus poderes y estructuras para afrontar esta enfermedad. La pandemia dejó de ser un problema del sector de la salud para convertirse en un fenómeno global, con esa mirada integradora tienen que afrontar la afectación desde los sectores sociales, económicos y políticos. Sin embargo, la realidad evidencia la desorganización en los sistemas públicos de salud, máxime cuando existen tantas políticas privatizadoras que han demostrado los crueles resultados vividos. Otro de los errores más comunes ha sido la falta de vigilancia de las estadísticas mundiales, ya sea por subestimar la pandemia o por no crear colocaciones necesarias para su enfrentamiento.

Además de la prevención, necesitamos de la adecuada previsión y que todos debemos ser conocedores de estas políticas en materia de prevención de riesgos y comprenderlas para ser capaces de acatarlas de forma inmediata y paliar así los riesgos lo más posible (Fernández, 2020, párr. 6).

Mientras más desigual sea un país en sus estructuras, "los grupos más vulnerables soportarán el peso de las repercusiones económicas 
de la pandemia y dispondrán de menos recursos para combatirla. Se debe prestar especial atención a las mujeres por su doble función de trabajadoras y cuidadoras" (Adhanom, 2020, parr. 9). Cada sociedad configura de manera peculiar su proceso de desarrollo, esa irrepetibilidad responde a criterios históricamente determinados sobre el orden social a proteger, los intereses sociales que predominan desde la hegemonía emanada de los grupos de poder y el particular esquema organizativo de su sociedad civil, entre otros. Precisamente la sociedad cubana ostenta determinadas características singulares en su experiencia sobre el control al sistema de salud y a partir de ellas haremos nuestra valoración.

Entre las potencialidades que propician el beneficioso crecimiento de la calidad en la salud se encuentra el desarrollo de las estructuras comunitarias, la capacidad y experiencia participativa de la sociedad civil en tareas sociales, y los actuales desarrollos cultural y educacional, entre otras. Precisamente la experiencia sociopreventiva y las capacidades de la sociedad permiten revisar la situación de forma integrada.

Cuba cuenta con un plan nacional aprobado por el Consejo de Ministros y dirigido por la máxima autoridad del país, que no solo integra al sistema de salud pública, sino a todos los organismos. El plan se basa en la contención al mínimo del riesgo de la introducción y propagación de la COVID-19 en el territorio nacional; así como la disminución de los efectos negativos que trae consigo una epidemia en la salud de la población y su impacto en la esfera económico-social (MSPa, 2020, parr. 11).

Se reorganizaron los servicios médicos ante la amenaza de la COVID-19 para evitar la afluencia innecesaria de personas a las instituciones sanitarias sin poner en riesgo la vida de los habitantes, apostando por las pesquisas activas efectuadas por el personal médico de conjunto con organizaciones de masas (Portal, 2020, párr. 3). El sistema de vigilancia está basado fundamentalmente en la estructura de la atención primaria de salud. Unido a ello se desarrolla un proceso de preparación de todo el personal de salud para que este sea capaz de evaluar a cada paciente e identificar los casos que puedan ser sospechosos de esta enfermedad. Se organizó un grupo de expertos de los centros de investigación científica con el objetivo de diseñar y ejecutar tareas de investigación e innovación para el desarrollo de nuevos conocimientos, productos y servicios relacionados con la COVID-19. 
La dirección del país ha decidido los centros y hospitales que serán utilizados territorialmente para el aislamiento y tratamiento de contactos, sospechosos y confirmados de la enfermedad, en este caso las universidades están siendo utilizadas en su rol social como centros de aislamiento, como parte del flujo de pacientes, desde su identificación como sospechoso y el tránsito en la institución designada; así como para los protocolos de tratamiento a seguir.

Se desarrolla un sistema de capacitación a profesionales, trabajadores y estudiantes del sector salud, así como de otros organismos de la administración central del Estado, organizaciones y trabajadores por cuenta propia vinculados al turismo (MSPa, 2020, párr. 8). Los cursos se apoyan en materiales docentes contextualizados al ámbito de desempeño o labor de cada trabajador de la salud para elevar el nivel de profundidad de la capacitación. El proceso de capacitación consta de varias etapas para garantizar la participación directa en la comunidad. Se cuenta, además, con un plan de comunicación informativo y preventivo.

Aunque el sistema de salud cubano presenta carencias e irregularidades, continúa apostando por el desarrollo técnico y profesional en aras de elevar la calidad de vida de toda la población, esencialmente desde la prevención.

Esta isla, pequeña, subdesarrollada, bloqueada, ha acondicionado lugares para cuarentena; ha desplegado un pesquisaje a toda la población con énfasis en las personas mayores; produce los medicamentos necesarios para su tratamiento; ha enviado misiones médicas a varios países para enfrentar el virus (Anaya, 2020, párr. 6), y continúa defendiendo que lo más importante es la vida humana.

\section{El cuidado de las personas mayores frente a la pandemia de la COVID-19}

La gerontología muestra una visión integral del desarrollo en el periodo final del ciclo vital humano y describe a las personas mayores como sujetos en pleno desarrollo psicológico, con potencialidades para el aprendizaje y para la diversificación de sus actividades cotidianas. Esta visión de la capacidad de desarrollo potencial debe primar en la labor 
de las personas que se encargan de la atención, cuidado y esparcimiento de los adultos mayores, en el nivel familiar o institucional, personas a las que denominamos cuidadores.

Esta figura resulta un problema de extraordinaria magnitud, que abarca múltiples perspectivas y en consecuencia requiere de respuestas pluridisciplinarias; se caracteriza por la multiplicidad de formas en la que se presenta hoy en nuestras sociedades.

La persona mayor tiene derecho a un sistema integral de cuidados que provea la protección y promoción de la salud, cobertura de servicios sociales, seguridad alimentaria y nutricional, agua, vestuario y vivienda; promoviendo que la persona mayor pueda decidir permanecer en su hogar y mantener su independencia y autonomía (OEA, 2015, p. 8).

La COVID-19 ha obligado al planeta a un confinamiento social no esperado. Para las personas mayores la realidad ha sido más impactante, por haber sido identificados como uno de los grupos de riesgos más sensible, tuvieron que acudir al cuidador. Por ello proponemos varios juicios organizativos de acuerdo con el ambiente o contexto espacial del adulto mayor:

- Cuidadores para personas mayores que viven en la unidad doméstica u hogar.

- Cuidadores para personas mayores a cargo del Estado (servicios sociosanitarios integrados).

- Cuidadores para personas mayores que viven solos, poseen su asistencia o seguridad social.

- Personas mayores desprotegidas totalmente.

\section{Cuidadores para personas mayores, que viven en la unidad doméstica u hogar}

Los cuidadores familiares son esenciales en el cuidado y bienestar de las personas mayores, resultan la solución ideal para las familias durante esta crisis sanitaria, en la mayoría de los casos se esmeran en asistir y proteger a sus seres queridos de cualquier riesgo que pueda enfermarlos. 
Se encargan de atenderlos para que el resto de los miembros de la unidad doméstica u hogar realicen sus actividades, dichos cuidadores son esencialmente del sexo femenino y su distribución parental oscila entre las hijas, las nueras y la esposa o esposo cónyuge.

Cuando la persona que cuida es el hijo, existe un vínculo natural familiar con la persona dependiente que favorece la disposición del cuidado, en la mayoría de las ocasiones, representa un fuerte impacto emocional para los hijos darse cuenta de que el padre, la madre o ambos ya no pueden valerse por sí mismos. Con esta situación de aislamiento social las medidas de cuidado tienen una creciente demanda por la labor más intensa de higiene, aseo y alimentación, que se traduce en un gasto económico no planificado. Gran parte de los cuidadores no pueden pensar en buscar un trabajo, pues las medidas extremas han sido en casi todos los sectores; además, no se relacionan con el exterior por miedo al contagio de ese adulto más vulnerable.

Los hijos e hijas de los cuidadores deben atender también a las necesidades de su familia y las propias; decidir dónde, cuándo y cómo invertir los esfuerzos y energías es difícil, sobre todo cuando la realidad requiere esfuerzo y sacrificio (de la Cuesta, 2013, p. 41).

Un caso especial es el de las nueras cuidadoras, aquí no existe un vínculo consanguíneo con la persona cuidada, solo media una relación de convivencia que no siempre está amparada por sentimientos de cariño o afecto hacia la persona que se cuida. Estas circunstancias pueden derivar en consecuencias negativas en la persona objeto del cuidado, conduciendo a malos tratos, carencia de afecto y desconfianza por la persona que cuida; quisiéramos precisar que no en todos los casos ocurre de la misma forma, existen nueras que cuidan con tanto amor y dedicación como el propio hijo o hija (de la Cuesta, 2013, p. 42).

El otro caso que se presenta es el del esposo o la esposa como cuidador. Cuando uno de los miembros de una pareja sufre un deterioro de la salud y necesita ayuda para sus actividades de la vida diaria, el cuidador principal suele ser el miembro de la pareja con mejor salud, la nueva situación que supone cuidar al esposo o a la esposa puede resultar difícil y exige una constante adaptación (de la Cuesta, 2013, p. 42). Una buena comunicación y actitud pueden ayudar mucho a rebajar estas tensiones, es más fácil aceptar la ayuda del esposo o la esposa, que la 
ayuda de familiares, amigos, vecinos o instituciones, en la medida en que se ve como una obligación matrimonial, así como una muestra de cariño por los años de convivencia juntos.

\section{Cuidadores para personas mayores a cargo del Estado (servicios sociosanitarios integrados)}

Los cuidadores profesionales de instituciones "son personas capacitadas a través de cursos teórico-prácticos de formación, dictados por equipos multi e interdisciplinarios de docencia, para brindar atención preventiva, asistencial y educativa al anciano y a su núcleo familiar" (Valderrama, 1997, p. 13), estas se encuentran en nuestro país en las instituciones de salud pública.

Los hogares para personas mayores, hogares de ancianos o residencias geriátricas resultan un entorno de gran vulnerabilidad por ser un lugar cerrado donde es difícil evitar el contacto entre los numerosos pacientes; en oportunidades no cuentan con el personal sanitario requerido. La persona responsable de los cuidados no debe tener factores de riesgo de complicaciones, será considerada contacto estrecho y deberá realizar cuarentena domiciliaria durante 14 días.

Actualmente hay en Cuba 155 hogares de ancianos con una disponibilidad de más de 11000 camas, y 294 casas de abuelos con régimen semiinterno. Para ser atendido en estas últimas es preciso tener 60 años o más, ser autónomo en sus actividades diarias, no poseer deterioro cognitivo avanzado ni tener alguna problemática social como que la familia trabaje y deje a la persona mucho tiempo sola o haber enviudado en un matrimonio de muchos años, algo que afecta en esas edades de manera especial (Barrios et al., 2020, párr. 17).

Este colectivo es también uno de los más proclives a adquirir el virus puesto que tienen necesidad de cuidados o asistencia, una preocupación especial por los que han sido excluidos de la sociedad y viven en la pobreza, con acceso limitado a los servicios de salud o en espacios de confinamiento como prisiones o asilos. La distancia social no debe convertirse en exclusión social (Kornfeld-Matte, 2020, parr. 17). 
En algunos países, estos servicios sociosanitarios integrados que otorgan "beneficios y prestaciones institucionales para responder a las necesidades de tipo sanitario y social de la persona mayor, con el objetivo de garantizar su dignidad y bienestar y promover su independencia y autonomía" (OEA, 2015, p. 4), se encuentran en alerta máxima, muchos se han sometido a una cuarentena total, han prohibido la entrada de visitantes, con la excepción de las situaciones del término de la vida, en otros se le permite a las familias sacar a sus abuelos de las residencias de mayores, temporalmente y con derecho y garantías de no perder su plaza, siempre y cuando se mantenga un aislamiento por seguridad, teniendo un carácter preventivo del contagio y humanitario al encontrarse con sus seres queridos.

\section{Cuidadores de personas mayores que viven solos, poseen su asistencia o seguridad social}

En el caso de los cuidadores profesionales domiciliarios, los asignados por las instituciones de seguridad y asistencia social, que pueden ser externos o internos y los cuidadores por beneficio contratados por la propia persona mayor o por un familiar del mismo, que incluyen dos variantes principales: los contratados por un salario y los contratados por la obtención de bienes materiales como: el disfrute de una vivienda, el uso de equipos electrodomésticos o medios de transporte, entre otros - esta última modalidad se utiliza con mucha frecuencia actualmente-.

En este segmento de la población se observa un incremento de hogares unipersonales, lo que a su vez aumenta la probabilidad de aislamiento social y soledad no deseada. Las estrictas medidas de confinamiento suponen un gran reto que desemboca en la ausencia o disminución de las interacciones sociales, que puede tener implicaciones para la salud física y mental (Lara, 2020, párr. 5).

En Cuba, con la asistencia de los trabajadores sociales, se investigó la situación de esas personas con la propuesta de insertarlas en el Sistema de Atención a la Familia y ayudarlos a higienizar sus domicilios; existen más de mil unidades gastronómicas destinadas a brindar los alimentos a hogares de personas mayores que viven solos y tienen dificultad para acudir a restaurantes, donde intervienen el Consejo 
de la Administración del Poder Popular, el Ministerio de Economía y Planificación y la Empresa de Comercio y Gastronomía unidos a líderes que identifican a estos grupos de poblaciones vulnerables (Martínez, 2020, párr. 6; Álvarez, 2020, párr. 3).

Esta atención como práctica diaria está caracterizada por una alta dosis de solidaridad y humanismo, que en los momentos de contingencia y aislamiento social en que vivimos constituye una fortaleza del sistema cubano ante la prevención del virus.

\section{Personas mayores totalmente desprotegidas}

Al evaluar esta contextualización, dudábamos, pues ¿quiénes serían los cuidadores de una persona que está totalmente desprotegida? La respuesta es una, la misma persona que debería recibir la atención tiene que cuidarse y cumplir el doble rol de sujeto-actor. Resultaba difícil la búsqueda pues no es la realidad de nuestro país; sin embargo, sí la de muchos hogares, por eso mantuvimos el análisis de esta en la clasificación.

Solo el 30 por ciento de las personas mayores en el mundo están cubiertas actualmente por programas de pensión, y en la mayoría de las sociedades, la salud, el empleo y los servicios de transporte no son fácilmente disponibles o accesibles. Ante este comportamiento, podrían incrementarse las dificultades económicas y financieras de las personas mayores. La gente que ha vivido con pobreza y desigualdad durante toda su vida llega a la vejez con profundas desventajas acumuladas (Stefanoni, 2013, párr. 2).

Su realidad es la de vivir y afrontar en soledad el aislamiento social y enfrentar dificultades para realizar tareas cotidianas con las medidas adoptadas; se encuentran en un riesgo adicional los que no tienen familiares o amigos cercanos (Huerta, 2020, párr. 12).

Es importante que los prestadores de asistencia sanitaria y la sociedad en su conjunto presten atención a las necesidades especiales de los grupos de población de edad mayor mediante las medidas siguientes: capacitación de los profesionales sanitarios, prevención y atención de 
las enfermedades crónicas que acompañan a la vejez, elaboración de políticas sostenibles sobre la asistencia a largo plazo y los cuidados paliativos y creación de servicios y entornos que favorezcan a las personas de edad (OMS, 2017, párr. 2).

La OMS está trabajando para proporcionar las estrategias, soluciones y suministros cruciales que los países necesitarán. Todos podemos apoyar a las personas mayores en estos tiempos. Muchos dependen de los servicios y apoyos que se les proporcionan en el hogar o en la comunidad para mantener su salud e independencia. Si bien es cierto que es importante el distanciamiento físico para evitar el contagio, también es importante comprender que no se trata de un aislamiento emocional con quienes o de quienes nos rodean (Adhanom, 2020, párr. 7; Orosa, 2020, párr. 3).

Tenemos que centrarnos en cómo hacer frente a este escenario tan difícil, para impedir también en lo posible que "el coronavirus cause más pobres, que muertos" (Ramonet, 2020, p. 45).

\section{Elementos configurativos de la figura del cuidador de la persona mayor}

Para adentrarnos en el estudio del cuidador, resulta vital analizar elementos que estructuran su representación, por ello desarrollaremos cada uno de ellos.

\section{Fundamentos de la labor del cuidador}

En este caso hacemos referencia a los cimientos, al sostén sobre el que recae esta tarea tan sacrificada, pero al igual tan digna de reconocimiento y ejemplo.

Consideramos que los pilares en que se sustenta el cuidado humano en la actualidad recaen principalmente en los motivos por los cuales una persona decide asumir el cuidado de su semejante, donde son imprescindibles los cuidados que se ofrecen, así como las necesidades y los derechos que surgen de los propios cuidados. 
Pero debemos valorar la satisfacción del cuidador, donde, a pesar de las dificultades y la dureza de la situación, los cuidadores manifiestan que cuidar a un familiar puede ser una de las experiencias más gratas y conmovedoras, independientemente de las numerosas tareas que ello implica; se pueden descubrir condiciones o aptitudes que probablemente de otra forma hubieran pasado desapercibidas.

\section{Motivación del cuidador}

El brindar cuidado es una labor que no cualquiera puede realizar; el cuidado trae consigo responsabilidades y cargas. No siempre esta labor es una decisión propia, en ocasiones no nos damos cuenta cuando estamos ofreciendo cuidados, pero poco a poco brindamos ayuda a otros para que puedan desarrollarse según las exigencias del entorno, hasta que un día reconocemos que sin nuestra ayuda no pueden convivir con el medio y es cuando tomamos la condición de cuidadores.

Somos del criterio que para ofrecer cuidados tiene que existir un consentimiento propio, pues esta labor tiene que ir aparejada de sentimientos afectivos hacia la persona que se cuida. No obstante, no siempre dichos sentimientos están presentes, son diversas las razones por las que un sujeto decide cuidar de una persona que se encuentra en una situación de dependencia; entre los diferentes motivos se encuentran los siguientes: motivación altruista, reciprocidad, motivos económicos, sentimientos de culpa, evitar la censura del entorno o por obligación -presiones familiares- (Montorio, 1999, p. 8). La mayoría de las razones por la que se cuida a una persona mayor llevan enraizadas fuertemente los valores morales y de obligación.

También se ha constatado que algunos de los cuidadores cuidan de la persona mayor para evitar la censura del entorno, por si el círculo de amistades con quien se desarrolla lo rechazarán por no cuidar de quien lo hizo con él.

Otras de las razones son por necesidades económicas, muchas de las personas que brindan cuidados obtienen mediante este empleo una remuneración que no encuentran en otra alternativa laboral. En otros casos viven como agregados con familiares y el cuidar de otra persona en su domicilio les brinda techo y comida, aparte de un salario. 
En otras ocasiones el hilo que motiva a una persona a cuidar de otra es un sentimiento de culpa, a partir de la existencia de dificultades o hechos en el pasado y se someten a cuidar al afectado en la actualidad buscando una compensación. Como otra incitación podemos valorar los móviles altruistas o por reciprocidad, no en todos los casos los cuidadores tienen un fin marcado, algunos conservan sentimientos de cariño, afecto y amor por la persona destinataria de los cuidados.

\section{Contenido elemental del cuidador}

Son múltiples las tareas que la persona en condición de cuidadora ofrece a otra que necesita de su ayuda para poder convivir con el entorno que la rodea y poder satisfacer las necesidades cotidianas que la vida les exige (Valderrama, 2019, párr. 13). Entre las labores más frecuentes que realizan los cuidadores se encuentran las siguientes: ayuda en las actividades domésticas, supervisión en la toma de medicamentos, auxilio para el transporte fuera del domicilio, colaboración en tareas de enfermería, ayuda para la higiene personal, el desplazamiento dentro del domicilio y resolver situaciones conflictivas derivadas del cuidado, y supervisión del cumplimiento de las medidas higiénicas, dietéticas y terapéuticas recomendadas en la aplicación de técnicas recreativas, deportivas y fisioterapéuticas.

\section{Necesidades de los cuidadores}

La variedad de tareas que deben asumir los cuidadores - responsabilidad y el compromiso que sobre ellos recae- trae como consecuencias que se vean alterados sus comportamientos habituales como resultado de la dedicación que implica el cuidado de las personas que lo precisan, dejando de lado su propia vida para dar paso a la vida de la persona de quien se cuida.

Dentro de las necesidades que con más frecuencia presentan los cuidadores podemos encontrar el disponer de tiempo libre, lo cual se convierte en casi una utopía por lo que no hay espacio para el ocio, la recreación ni el contacto con la sociedad, lo que se convierte en una insuficiencia vital, por ello se olvidan de sí mismos. 
Otra insuficiencia que manifiestan los cuidadores es el apoyo en el desarrollo de diferentes labores en el hogar, principalmente en las mujeres cuidadoras, porque sobre ellas recaen las labores hogareñas, lo que hace que se produzca una sobrecarga. También se encuentra el beneplácito que se reconozca la labor que desempeñan y no se le imponga, la familia debe ayudar a brindar cuidados y convertirse también en gestora de ellos. La falta de recursos económicos resulta ser otra demanda importante e insatisfecha, ya que en oportunidades el cuidador deja su trabajo o actividades generadoras de ingresos para poder cuidar de su familia y a su vez los gastos aumentan por las necesidades propias de la persona dependiente.

Resulta de gran importancia que los cuidadores puedan satisfacer sus necesidades y se vean apoyados por la familia. Por ser esta la principal red de apoyo es la encargada directa de brindar cuidados y satisfacer demandas básicas, es muy fácil delegar labores ante la sensación de impotencia y desconcierto que se vive respecto a la situación de envejecimiento de un miembro de la familia, pero estas deberían ser conjuntas para evitar en el cuidador el síndrome de burnout (Castro et al., 2009, p. 35). Con el apoyo familiar no solo se beneficia la persona en situación de dependencia, sino que disminuye la carga de responsabilidades de la persona que ofrece cuidados.

\section{Derechos de los cuidadores}

El brindar cuidado es una labor que exhibe mucho esmero y dedicación, lo que conduce a una difícil situación para aquellos individuos que la ofrecen. Como resultado de la afectación de la calidad de vida por la que atraviesan los cuidadores, brindaremos un conjunto de derechos a los que estos tienen acceso: dedicar tiempo y actividades sin sentimientos de culpa, experimentar sentimientos negativos por ver al enfermo o estar perdiendo a un ser querido, resolver aquello que son capaces y el derecho a preguntar sobre aquello que no se comprenda, buscar soluciones que se ajusten a sus necesidades y a las de los seres queridos, ser tratados con respeto por aquellos a quienes se les solicita consejo y ayuda, y seguir la propia vida (Austidillo \& Mendinueta, 2018, párrs. 6-7). 
diseñar medidas de apoyo a las familias y los cuidadores mediante la introducción de servicios para quienes realizan la actividad de cuidado de la persona mayor, teniendo en cuenta las necesidades de todas las familias y otras formas de cuidados, así como la plena participación de la persona mayor, respetándose su opinión (OEA, 2015, p. 8).

Teniendo en consideración la forzosa situación por la que atraviesan los cuidadores en el espacio social y psicológico, resultan de gran apoyo los derechos anteriormente aludidos; estos se constituyen en vías que posibilitan que se sientan aliviados con la carga emocional y laboral que llevan.

\section{Protección jurídica de la figura del cuidador de la persona mayor en Cuba}

Unido a los altos niveles de envejecimiento de la población discurre la figura del cuidador de las personas mayores, por lo que resulta de vital importancia garantizarle, desde el punto de vista jurídico, la protección de sus derechos. Con ese objetivo realizamos una valoración en el ámbito del derecho en nuestro país. El análisis partió de la Constitución de la República, seguidamente en el Código de Trabajo y la Ley de Seguridad Social.

\section{La protección al cuidador en la Constitución de la República de Cuba}

Con el ánimo de realizar un somero análisis de la actualidad sobre la protección jurídica de los cuidadores, debemos comenzar por la Constitución de la República que como Ley fundamental define los principios de protección para toda la población, incluido el grupo etáreo objeto de esta investigación. En su artículo 68, se aprecia que el Estado protege a los abuelos u otros familiares, en función del cuidado y la atención (Asamblea Nacional del Poder Popular en Cuba, 2019, p. 5). 
Por su parte, el artículo 84 de la Ley de leyes aborda la obligación por parte de los hijos de respetar, atender y proteger a sus madres, padres y otros parientes, lo que ayudará en su calidad y esperanza de vida. El artículo 88 señala que

Estado, la sociedad y las familias, en lo que a cada uno corresponde, tienen la obligación de proteger, asistir y facilitar las condiciones para satisfacer las necesidades y elevar la calidad de vida de las personas adultas mayores. De igual forma, respetar su autodeterminación, garantizar el ejercicio pleno de sus derechos y promover su integración y participación social (ANPP, 2019, pp. 6-9).

Dado al nivel de envejeciendo de la población y por ende su proclividad a situaciones de riesgo, resulta necesaria la inclusión del cuidador en el texto legal, que aún no se identifica explícitamente como la figura que puede atender, cuidar y proteger a este grupo etáreo. Desde nuestra Carta Magna se otorga la responsabilidad de atender a las personas mayores en dos aristas de análisis: la familia y el Estado, sin incluir la realidad familiar de muchos contextos.

\section{La protección al cuidador en el Código de Trabajo}

Resulta de capital importancia el enfoque jurídico que provee el Código de Trabajo a los cuidadores para la protección de las personas mayores, un enorme reto ante el envejecimiento poblacional. Solamente existe un artículo que aborda la atención a este grupo etáreo mediante las licencias no retribuidas a trabajadores con responsabilidades familiares. El artículo 108 señala que "en el sector estatal a solicitud del trabajador, el jefe de la entidad puede conceder licencias no retribuidas a trabajadores con responsabilidades familiares para su atención y cuidado" (ANPP, 2013, p. 19).

Evidente resulta el insuficiente respaldo legislativo en la ley laboral que consolida y perfecciona las regulaciones que garantizan la protección de los derechos y el cumplimiento de los deberes ante una necesidad creciente y real, lo cual entorpece el crecimiento de esta figura ocupacional. 


\section{La protección al cuidador en la Ley de Seguridad Social}

En nuestro país se encuentran vigentes la Ley 105/08 de Seguridad Social y su reglamento, el Decreto Ley 203/08 (ANPP, 2008). Ambos cuerpos legales mantienen el reconocimiento de la asistencia social a domicilio, servicio que persigue brindar apoyos complementarios, de carácter personal y social, en el domicilio de personas que vivan solas y cuya discapacidad les impide el desempeño independiente de las actividades de la vida diaria. De esta manera, se logra que las personas mayores o personas con discapacidad, que viven solas, carecen de familiares $\mathrm{u}$ otras personas convivientes con ellos e imposibilitadas de realizar las actividades de la vida diaria de manera autónoma, reciban protección en su propio medio y satisfagan sus principales necesidades biológicas, psicológicas, comunicativas y sociales.

El asistente social a domicilio, también llamado cuidador, ha de desempeñar diversas funciones, las cuales no han sido normadas, pero sí definidas en una metodología del Ministerio de Trabajo y Seguridad Social (MTSS) que indica los procedimientos para el otorgamiento, ejecución y revisión de las prestaciones de la asistencia social.

Son incuestionables los logros alcanzados por el país en materia de seguridad y asistencia social; en consecuencia, los beneficios que por estos conceptos reconoce el ordenamiento jurídico se han ido modificando, valorando el servicio de asistencia social a domicilio, pese a las dificultades existentes en su concepción, como las cuestiones sobre las funciones del cuidador y el control de su cumplimiento.

En principio, resulta comprensible que el Estado no debe cargar al presupuesto de la asistencia social el cuidado de aquellas personas que, por razón de la edad, dado el grado de dependencia que poseen, se encuentran requeridos de un asistente social a domicilio teniendo un familiar a quien le corresponde asumir dicha responsabilidad.

De lo anterior se deduce la imperante realidad de un gran número de cuidadores informales o no oficiales, que ofrecen cuidados a personas adultas dependientes y no poseen un respaldo legal. Es conveniente formalizar la situación de los cuidadores no designados por el servicio de asistencia social y dotarlos de beneficios, atenciones y garantías como 
consecuencia de su carga, pues tanto los formales como los informales desarrollan la misma actividad.

Tomando como base la idea anteriormente abordada, surge la necesidad de la inclusión de los cuidadores informales en nuestras normas jurídicas con el objetivo de formalizar y recoger legalmente su estatus social, situación que le concede un conjunto de garantías actualmente no previstas en ningún ordenamiento jurídico.

Bajo el prisma de las ciencias jurídicas se abre un abanico de interrogantes relacionados con la necesaria corresponsabilidad del Estado y la familia en el cuidado de este grupo etáreo, máxime de aquellos que presentan un alto grado de vulnerabilidad, ya sea porque viven solos, padecen de enfermedades que aceleran el deterioro físico-mental, han adquirido con la edad alguna discapacidad, reciben poco o ningún apoyo familiar o se encuentran en estado de abandono (León, 2010, p. 65).

La atención y los cuidados ante el envejecimiento poblacional son un hecho que sin duda se ha convertido en una problemática tanto social como jurídica, situación que nos obliga de forma inmediata a buscar vías y estrategias integrales para su solución.

\section{Conclusiones}

El estudio doctrinal de la figura del cuidador evidencia la existencia de diversas definiciones conceptuales y teóricas. Nuestra definición se ajusta a considerarlo desde dos ópticas diferentes: el cuidado informal fundado en una relación social basada en afecto, cariño y servicio que se realiza a través de una relación de parentesco, vecindad o amistad, y el cuidado formal previsto por asistentes y enfermeras, entre otras.

La pandemia de COVID-19 ha demostrado la vulnerabilidad de las personas mayores de agravarse al contraer la enfermedad. La mayoría de los Estados como parte del propio proceso de enfrentamiento no implementaron oportunas políticas de prevención de riesgos ni acciones integrales de sus estructuras para afrontar y reducir este flagelo. En el caso específico de Cuba se aprobó un plan de acciones que tiene su fundamento en la contención del virus, la disminución de los efectos negativos en la salud de la población, con énfasis en este grupo de 
riesgo y su impacto en la esfera económico-social, demostrando cada día la coherencia de su estrategia de enfrentamiento.

Estructuramos las clasificaciones de los cuidadores desde un criterio socioecológico destinado a organizar la atención de la persona mayor de acuerdo con el ambiente o contexto espacial de ocurrencia, en función de lo cual reconocemos la existencia de cuatro juicios organizativos de análisis: cuidadores para personas mayores que viven en la unidad doméstica u hogar, cuidadores para personas mayores a cargo del Estado (servicios sociosanitarios integrados), cuidadores para personas mayores que viven solos pero poseen su asistencia o seguridad social y personas mayores totalmente desprotegidas.

El cuidador de la persona mayor necesita ser potenciado desde una visión multidisciplinaria que incluya tanto la regulación informal como formal. El análisis al problema estudiado debe sustentarse en un enfoque victimocriminológico central, perspectiva que implicaría la contribución de otras disciplinas sociales como la sociología, la medicina y la psicología social, entre otras, mientras la regulación formal se materializa en los diversos enfoques jurídicos de amparo de esta figura. En el caso de Cuba, la variante formal concretada en la protección jurídica nos muestra que no existe un reconocimiento legislativo al cuidador ni mecanismos institucionales implementados.

\section{Referencias}

Adhanom Ghebreyesus, T. (2020). Alocución de apertura del Director General de la OMS en la rueda de prensa sobre la COVID-19. https://www.who.int/es/ $\mathrm{dg} /$ speeches/detail/who-director-general-s-opening-remarks-at-the-ediabriefing-on-covid-19

Álvarez Palacio, D. (23 de abril de 2020). Ante la Covid-19: Atención al adulto mayor en una zona en cuarentena. Periódico Granma. http://www. granma.cu/fotorreportajes/2020-04-23/ante-la-covid-19-solidaridad-yhumanismo-23-04-2020-18-04-18

Alvero Francés, F. (1978). Diccionario Cervantes. Manual de la Lengua Española. Editorial Pueblo y Educación. 
Anaya Cruz, B. (2020). Coronavirus, sistema de salud y Economía en Cuba. https:// www.cubainformacion.tv/cuba/20200403/85371/85371-coronavirussistema-de-salud-y-economia-en-cuba

Austudillo, W., \& Mendinueta, C. (2018). Derechos de los enfermos y Cuidadores. http://www.indesol.gob.mx/web/Index.php?1=12

Barrios, M., Hernández Porto, Y., Rodríguez Roque, N., García Santos, N., Brunet Portela, L., E Pimentel Miranda, Z. (22 de marzo 2020). Mi hogar, mi refugio. Periódico Digital Juventud Rebelde. http://www.juventudrebelde. cu/cuba/2020-03-22/mi-hogar-mi-refugio

Castro Espejo, R. E., Solórzano Gil, H. P., E Vega Chávez, E. P. (2009). Necesidades de cuidados que tienen los cuidadores de personas en situación de discapacidad por parte de su familia en la localidad de Fontibón. (Tesis de grado, Pontificia Universidad Javeriana, Bogotá,).

Cruz Rivas Herrera, J., E Ostiguin Meléndez, R. M. (2016). Cuidador: ¿concepto operativo o preludio teórico? Revista Enfermería Universitaria, 8(1).

Cuba, Asamblea Nacional del Poder Popular. Ley 105, de Seguridad Social y su Reglamento, 27 de diciembre 2008.

Cuba, Asamblea Nacional del Poder Popular, (2013). Ley 116 del 20 de diciembre, Gaceta Oficial 29.

Cuba, Asamblea Nacional del Poder Popular, (2019). Constitución de la República de Cuba.

Cuba, Ministerio de Salud Pública de la República. (2020a). Para prevenir y enfrentar el nuevo coronavirus, la participación popular es esencial. https:// salud.msp.gob.cu/?p=4023

Cuba, Ministerio de Salud Pública de la República. (5 de mayo de 2020b). Parte de Cierre del 4 de mayo 2020. https://salud.msp.gob.cu/

de la Cuesta Benjumeda, C. (2013). El cuidado del otro: Desafios y posibilidades. Editorial Alianza.

Devandas, C. (2019). Es inaceptable segregar a una persona por ser mayor y tener una discapacidad. https://www.gerontologia.org/portal/information/ showInformation.php?idinfo $=4405$

Fernández Moreno, S. A. (2 de abril 2020). Prevenimos problemas o ayudamos a solucionarlos. http://www.legaltoday.com/practica-juridica/social-prevencionriesgoslaborales/referencias-al-covid-19

Gallardo, P., E Rojas, M. (2016). El rol del cuidador en el adulto mayor. (Tesis de grado, Universidad Nacional de Cuyo, Argentina).

Guimaráes, M. (2015). Aspectos psicológicos en cuidadores formales. (Tesis de doctorado, Universidad de Madrid, España). 
Hadar, Y. (20 marzo 2020). El número de pobres en la América Latina puede crecer en 35 millones por el coronavirus COVID-19. Noticias ONU. https:// news.un.org/es/story/2020/03/1471522

Huerta Mendoza, 1. (30 de marzo 2020). Consecuencias de aislar a los ancianos durante la pandemia de COVID-19. Gaceta UNAM. https://www. gaceta.unam.mx/aislar-a-los-ancianos-durante-la-pandemia-reduciria-latransmision-de-covid-19/

Kornfeld-Matte, R. (2020). Es inaceptable el abandono de personas mayores en los asilos durante la pandemia del coronavirus. http://www.radiobayamo.icrt. cu/2020/03/28/es-inaceptable-el-abandono-de-personas-mayores-en-losasilos-durante-la-pandemia-del-coronavirus/

Lara Pérez, E. (2020). COVID-19: Cómo ayudar a las personas mayores que viven solas. https://theconversation.com/covid-19-como-ayudar-a-las-personasmayores-que-viven-solas-133663

León García, L. (2010). La pluralidad del régimen tuitivo de los adultos mayores ante el deterioro de la capacidad física y mental. (Tesis de especialización, Universidad Central Marta Abreu de las Villas, Cuba).

Martínez Zelada, L. M. (25 marzo 2020). Prioriza comercio atención a adultos mayores solos, ante pandemia. Agencia cubana de noticias. http://www.acn. cu/cuba/62490-prioriza-comercio-atencion-a-adultos-mayores-solos-antepandemia

Montorio Cerrata, I., Fernández de Trocóniz, M. I., E Díaz Veiga, P. (1999). Cuando las personas mayores necesitan ayuda. Revista Guía para cuidadores y familiares, 3(1).

Navarrete Calderón, C. (2004). Criminología: alcances, ciencia, disciplina y práctica social. En T. de Armas Fonticoba (Coord.), Criminología (pp. 2-34). Editorial Félix Varela.

Oficina Nacional de Estadísticas e Información. (2018). Anuario Estadístico de Cuba, Población.

Organización de Estados Americanos. Convención interamericana sobre la protección de los derechos humanos de las personas mayores (A-70), (15 de diciembre de 2015).

Organización Mundial de la salud. (2017). La salud mental y los adultos mayores. https://www.who.int/es/news-room/fact-sheets/detail/la-salud-mental-ylos-adultos-mayores

Organización Mundial de la Salud. (2018). Informe Mundial sobre la violencia y la salud. 
Orosa Fraíz, T. (28 marzo 2020). Las personas mayores ante la COVID-19. Cubadebate. http://www.cubadebate.cu/especiales/2020/03/28/las-personasmayores-ante-la-covid-19/\#.Xq4oxHJuncc

Pérez Cabrera, F. (2016). Cuidadores de ancianos profesionales. Editorial Pueblo y Educación.

Portal Miranda., J. A. (2020). Reorganiza Cuba su sistema de salud para hacer más efectiva la lucha contra el coronavirus. http://www.radiohc.cu/noticias/ salud/217731-reorganiza-cuba-su-sistema-de-salud-para-hacer-mas-efectivala-lucha-contra-el-coronavirus

Ramonet, I. (2020). Ante lo desconocido. La pandemia y el sistema-mundo. http:// www.cubadebate.cu/especiales/2020/04/25/especial-de-ignacio-ramonetante-lo-desconocido-la-pandemia-y-el-sistema-mundo/

de los Reyes, M. C. (2008). Construyendo el concepto del cuidador de ancianos. Foro de Investigación, Envejecimiento de la población en Mercosur, Curitiba, Brasil.

Stefanoni, S. (2013). Los mejores (y los peores) países para ser viejo. https:// www.bbc.com/mundo/noticias/2013/10/130726_salud_indice_paises_vejez_men

Valderrama, H. R. (1997). Manual argentino para el cuidado de ancianos. Editorial Imprenta Lux.

Valderrama, H. R. (2019). Cuidadores de ancianos profesionales. En Cursos de Formación Multimedial Online Gerontología. https://www.cuidadoresdeancianos.com/cuidadores/cuidadores-profesionales/ 